\title{
A Multilayer LSTM Auto-Encoder for Fetal ECG Anomaly Detection
}

\author{
Inna SKARGA-BANDUROVA ${ }^{\mathrm{a}, 1}$, Tetiana BILOBORODOVA ${ }^{\mathrm{b}}$, Illia SKARHA- \\ BANDUROV ${ }^{\mathrm{c}}$, Yehor BOLTOV ${ }^{\mathrm{b}}$, Maryna DERKACH ${ }^{\mathrm{d}}$ \\ a School of Engineering, Computing and Mathematics, Oxford Brookes University \\ ${ }^{\mathrm{b}}$ G.E. Pukhov Institute for Modelling in Energy Engineering \\ ${ }^{\mathrm{c}}$ Luhansk State Medical University \\ d Volodymyr Dahl East Ukrainian National University
}

\begin{abstract}
The paper introduces a multilayer long short-term memory (LSTM) based auto-encoder network to spot abnormalities in fetal ECG. The LSTM network was used to detect patterns in the time series, reconstruct errors and classify a given segment as an anomaly or not. The proposed anomaly detection method provides a filtering procedure able to reproduce ECG variability based on the semi-supervised paradigm. Experiments show that the proposed method can learn better features than the traditional approach without any prior knowledge and subject to proper signal identification can facilitate the analysis of fetal ECG signals in daily life.
\end{abstract}

Keywords. Auto-encoder, long short-term memory (LSTM), anomaly detection, fetal electrocardiography (ECG)

\section{Introduction}

The modern wearable ECG recorders can store a large amount of information, which allows recording throughout the day, tracking changes with high resolution and data transmission. In addition, the analysis of this data based on machine learning models using computational algorithms makes it possible to detect changes in a timely manner and personalize treatment. At the same time, the use of wearable and intelligent devices for telemedicine and outpatient care requires accurate and reliable analysis of biomedical signals in real-time, the study of their characteristics and the corresponding physiological events.

In this context, we consider the problem of detecting abnormalities in the fetal ECG obtained from a portable device attached to the mother's abdomen, which allows longterm monitoring during pregnancy. Fetal ECG reflects the electrophysiological activity of the fetal heart. Due to the fact that the size of the maternal ECG detected in the abdominal cavity is approximately 2-10 times the size of the fetal ECG [1], the analysis of abnormalities in the fetal ECG signal is still challenging. Moreover, it needs to detect fetal QRS complex in a very noisy environment [2] and analyze the huge amount of collected data. The process is therefore expensive and time-consuming. It is

${ }^{1}$ Corresponding Author: Inna Skarga-Bandurova, School of Engineering, Computing and Mathematics, Oxford Brookes University, Wheatley Campus, Oxford, UK; E-mail: iskarga-bandurova@brookes.ac.uk. 
advantageous to characterize data in advance on ECG states and thereby use consequent deep analysis only if needed.

Any change in the standard ECG signal leads to heart disease (abnormal). Based on this statement, differentiating the ECG into normal and abnormal will help reduce the time it takes experts to interpret the ECG. Expert analysis will be necessary only if there are deviations in the signal from the normal ECG.

Different approaches are used to detect deviations in the ECG signals. In [3], the novel S-transform and frequency division technique was proposed to analyze the timefrequency sub-band matrices stemming from the ECG signals. The autoregressive timefrequency analysis was applied in [4] for finding the coefficients and time-frequency description of the ECG signal. The study [5] proposed a segmented-beat modulation method as a new template-based filtering procedure able to reproduce ECG variability. A model that utilizes long short-term memory (LSTM) prediction errors as input features for the second stage of anomaly class prediction was proposed and described in [6]. The results show that the error vectors represented by their summary features carry useful predictive information about the type of the ECG anomaly. In [7], a deep learning model based on residual network (ResNet) that adopts the 1-D octave convolution was proposed to extract fetal ECG. The study [8] proposed a cardiac abnormality detection algorithm for optical photoplethysmography (PPG) based on an autoencoder. The autoencoder was trained to recognize typical normal PPG morphology and rhythm.

This article aims to extend these studies and describe a multilayer LSTM autoencoder network to obtain information about changes or abnormalities in the ECG during fetal monitoring via a non-invasive wearable device. Following [9], the LSTM-based encoder is used to detect deviations and assess the presence of abnormalities in the fetal ECG rhythm as well as to help determine the need to visit a doctor between regular schedules for a more detailed and comprehensive examination.

\section{Methods}

In this study, we tested a semi-supervised learning approach to assess ECG abnormalities using the LSTM autoencoder. The key idea is to learn an LSTM autoencoder on a multivariate ECG signal to perform binary classification. This can be achieved in the following way. The LSTM autoencoder is trained on ECG data with normal rhythm and on new reconstructed data. Then the specific threshold is applied, and the loss function is calculated. If the loss is higher than the threshold, it is defined as an anomaly. The defined anomaly is recorded and counted, and if the recording is higher than the threshold, it is triggered to warn the patient to visit a doctor for a more thorough examination.

Further, the description of the proposed methodology is presented. A semisupervised learning pipeline to detect abnormalities in the ECG using the LSTM autoencoder includes the following main stages: data preprocessing, model training, anomaly detection.

\subsection{Methodology}

Traditionally, preprocessing includes ECG signals normalization and segmentation. Since we used normalized data, the normalization stage was skipped. The sliding window is used for signal segmentation. The waveform divides into overlapping segments. This approach is used to get instances of waveform shape. The necessary primary condition 
in this model is the size of the window. A window is represented as a shape of consecutive data elements per time unit. The result of signal segmentation is the data set of ECG shapes.

In situations when some labels are unavailable for one of the classes, for example, when negative class in unlabeled, it is possible to utilize these unlabeled data drawn from marginal density [10]. In case of binary classification, having one labeled class and set of unlabeled data the classifier can be trained using only one class (for example, positive) and unlabeled data.

The classifier should discriminate between positive class and unlabeled data. However, a naive attempt to classify positive class and unlabeled data in many cases lead to bias. To address this challenge, we utilize unlabeled data to evaluate the risk for negative samples [11] and introduce the indicator function defined as binary values $(0,1)$, which takes the value of 0 if the predicted class is positive and 1 if the predicted class is negative.

The anomaly detection is performed using the L1 Loss function. The L1 Loss or Mean Absolute Error (MAE) is used for model evaluation. MAE is the sum of absolute differences between the true value and predicted values that measures the average magnitude of errors in a set of predictions, without considering their directions.

$$
\text { L1LossFunction }=\sum_{i=1}^{n}\left|y_{\text {true }}-y_{\text {predicted }}\right|
$$

The ECG abnormalities can be assessed based on the following assumptions. The anomaly shapes that represent the segment $S$ follow the Boolean function $\{0,1\}$

$$
f(S)=\left\{\begin{array}{l}
1, \text { if the anomaly is detected } \\
0, \text { otherwise }
\end{array}\right.
$$

where $f(S)$ is a function of segment anomaly estimation.

Abnormalities assessment of ECG signal $\alpha$ is defined as a function $g(\alpha)$ that calculated as a percentage of detected anomaly segments in ECG signal

$$
g(\alpha)=\frac{\sum_{j=1}^{w_{i}}\left(f\left(S_{i}^{j}\right)\right)}{w_{i}}
$$

where $\alpha$ is an ECG signal, $w_{i}$ is a number of all segments in ECG signal $\alpha, f\left(S_{i}^{j}\right)$ is a Boolean function defined in (2).

Further, if a number of anomaly segments is higher than the defined threshold, ECG signal $\alpha$ will be assessed as abnormal.

\subsection{Experimental Data}

For the experiment stage, we used open-source data [12] from PhysioNet [13]. In this dataset, non-invasive fetal ECG recordings were collected from pregnant women during routine medical visits. The data include recordings with diagnosed fetal arrhythmias by echocardiography, and equal control recordings diagnosed with a normal rhythm. All data were acquired at a sampling frequency of $500 \mathrm{~Hz}$ (ARRs 6-9, Table S1) or $1000 \mathrm{~Hz}$ 
(ARRs 1-5 and 10-11 and all NRs) and with a 16-bit resolution and a range of $\pm 8 \mathrm{mV}$. Data were recorded continuously.

Each entry consists of four or five abdominal canals that correspond to the fetus ECG: Abdomen_1, Abdomen_2, Abdomen_3, Abdomen_4, Abdomen_5, and one channel that corresponds to the highlighted mother's ECG: ECG. The entries are annotated as follows: ARR - arrhythmia fetus, NR - normal rhythm fetus. Records NR 01 and ARR 01 were used in the experiment.

A fragment of the ARR 01 includes six records (see Fig. 1). The upper signal corresponds to maternal ECG, five lower signals correspond to fetal ECG.

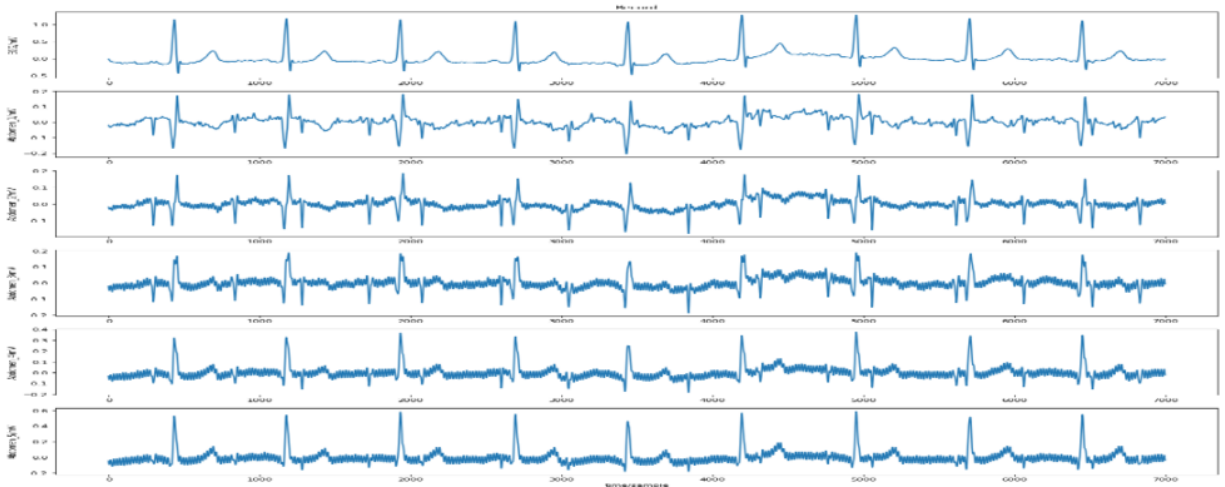

Figure 1. The fragment of data.

For the experiment, we previously removed the maternal ECG from the studied ECG signals [14].

\section{Results}

Segmentation was carried out using a sliding window with a size of 550 epochs. The offset is $1 \%$, i.e. 6 epochs. The model was trained using two configurations of LSTM auto-encoders. The model was trained on segmented fetal data with a normal rhythm. The number of epochs is 350 for the experiment setup. The architecture of used classifiers is shown in Table 1.

Table 1. The parameters of the LSTM classifiers.

\begin{tabular}{rrrrrrrr}
\hline Parameters & $\begin{array}{c}\text { LSTM } \\
\text { cells }\end{array}$ & $\begin{array}{c}\text { Network } \\
\text { layers }\end{array}$ & Optimizer & $\begin{array}{c}\text { Dropout } \\
\text { /Dense }\end{array}$ & Epoch & $\begin{array}{c}\text { Batch } \\
\text { size }\end{array}$ & $\begin{array}{c}\text { Cost } \\
\text { function }\end{array}$ \\
\hline Default settings & 64 & 4 & NAdam & 0 & 350 & 128 & Focal Loss \\
Our settings & 80 & 5 & Adam & 1 & 250 & 128 & L1Loss \\
\hline
\end{tabular}

For the second classifier, we increased the number of the LSTM layers to 5 resulting in that the network learned features at different time scales.

Anomalies were identified by classifying abdominal lead data that correspond to segmented fetal ECG data, which are annotated as ARR. The control data used $15 \%$ of the segmented fetal ECG dataset with a normal rhythm, which was not used in training. The threshold value for the loss function, which indicates a prediction error. All values 
of loss function above the threshold are flagged as an anomaly. If the loss function is below the threshold, it was classified as a normal heartbeat.

The visualization of original and reconstructed normal and anomalous ECG segments is shown in Fig. 2.

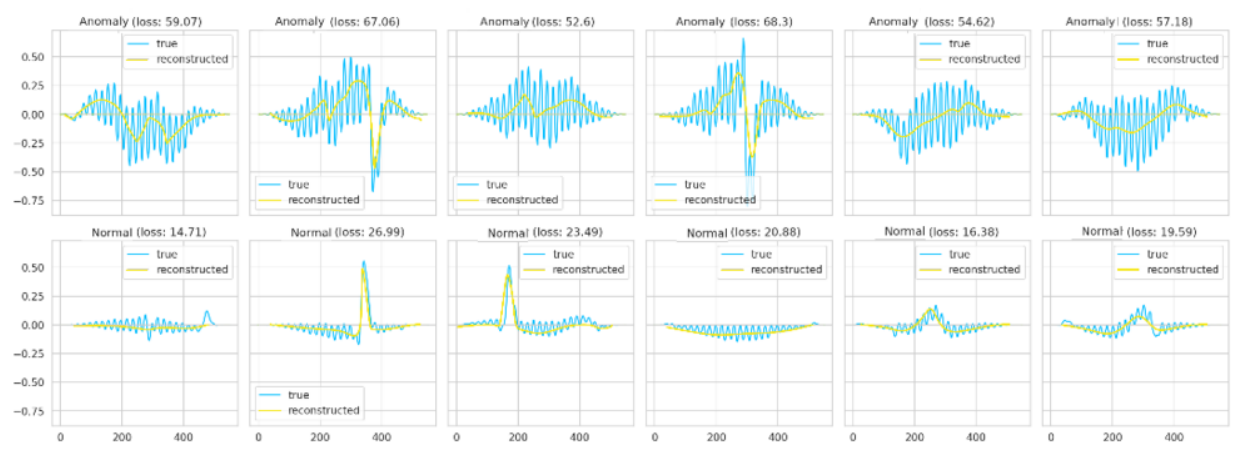

Figure 2. The examples of simulated data with detected anomalies.

The resulting threshold value for the loss function, which indicates a prediction error, was defined to be 52. All values of loss function above this value are flagged as an anomaly. If the loss function is below the threshold, we classify it as a normal heartbeat. As noted earlier, exceeding the threshold could be a signal to see a doctor for a more specialized examination.

\section{Conclusion}

In this work, we used LSTM auto-encoders to assess the presence or absence of features that determine the normalcy of the signal. Auto-encoders have made it possible to reduce the multidimensional problem of whether an ECG segment matches the patterns that define normality in just two dimensions: reconstruction errors in the time and frequency domains. A detailed study of the obtained results of detecting ECG signal anomalies showed that the recordings alternate segments of low and high quality, and the presence of an anomaly affects the likelihood that the following segments will be abnormal. The neural networks make it possible to analyze the ECG signal without additional data processing; however, more accurate detection is needed for additional signal processing in order to identify the QRS-complex elements used for further analysis [15]. There are at least three benefits of the proposed methodology: (i) feature extraction, and selection techniques are not needed, (ii) the proposed approach can help to inform about significant abnormalities, (iii) it is possible to predict ECG anomaly using only one class of labelled data. One more positive effect of this approach is that the final anomaly estimate has a certain range at $(0,1)$ and is clearly interpreted as the probability that the segment is an anomaly, which would not be so unambiguous if we were left with the reconstruction error as an anomaly estimate. 


\section{References}

[1] Ungureanu GM, Bergmans JWM, Oei SG, Ungureanu A, Wolf W. The event synchronous canceller algorithm removes maternal ECG from abdominal signals without affecting the fetal ECG, Computers in Biology and Medicine 2009; 39(6): 562-567.

[2] Yuen B, Dong X, Lu T. Detecting Noisy ECG QRS Complexes Using Wavelet CNN Autoencoder and ConvLSTM, in IEEE Access, 2020; 8: 143802-143817, doi: 10.1109/ACCESS.2020.3012904.

[3] Tripathy RK, Paternina MR, Arrieta JG, Zamora-Méndez A, Naik GR. Automated detection of congestive heart failure from electrocardiogram signal using Stockwell transform and hybrid classification scheme. Computer methods and programs in biomedicine, 2019; 173: 53-65.

[4] Gupta V, Kanungo A, Kumar P, Sharma AK, Gupta A. Auto-regressive Time Frequency Analysis (ARTFA) of Electrocardiogram (ECG) signal. International Journal of Applied Engineering Research, 2018; 13(6): 133-138.

[5] Agostinelli A, Sbrollini A, Giuliani C, Fioretti S, Nardo FD, Burattini L. Segmented beat modulation method for electrocardiogram estimation from noisy recordings. Medical Engineering \& Physics, 2016; 38(6): 560-568. doi:10.1016/j.medengphy.2016.03.011

[6] Chauhan S, Vig L, Ahmad S. ECG anomaly class identification using LSTM and error profile modeling. Comput Biol Med. 2019 Jun;109:14-21. doi: 10.1016/j.compbiomed.2019.04.009.

[7] Vo K, Le T, Rahmani AM, Dutt N, Cao H. An Efficient and Robust Deep Learning Method with 1-D Octave Convolution to Extract Fetal Electrocardiogram. Sensors (Basel). 2020 Jul 4; 20(13), 3757. doi: $10.3390 / \mathrm{s} 20133757$.

[8] Whiting S, Moreland S, Costello J, Colopy G, McCann C. Recognising Cardiac Abnormalities in Wearable Device Photoplethysmography (PPG) with Deep Learning. arXiv preprint, 2018, arXiv:1807.04077.

[9] Hou B, Yang J, Wang P, Yan R. LSTM-Based Auto-Encoder Model for ECG Arrhythmias Classification, IEEE Transactions on Instrumentation and Measurement, 2020; 69(4): 1232-1240, doi: 10.1109/TIM.2019.2910342.

[10] Sakai T, du Plessis MC, Niu G, Sugiyama M. Semi-Superised Classification based on classification from positive and unlabelled data, https://arxiv.org/abs/1605.06955.

[11] du Plessis MC, Niu G, Sugiyama M. Analysis of learning from positive and unlabeled data. In NIPS, pp. 703-711, 2014., du Plessis, M. C., Niu, G., and Sugiyama, M. Convex formulation for learning from positive and unlabeled data, In ICML, 37, (2015), 1386-1394.

[12] Behar JA, Bonnemains L, Shulgin V, Oster J, Ostras O, Lakhno I. Noninvasive fetal electrocardiography for the detection of fetal arrhythmias. Prenat Diagn. 2019 Feb;39(3):178-187. doi: 10.1002/pd.5412.

[13] Goldberger AL, Amaral LAN, Glass L, Hausdorff JM, Ivanov PC, et al. PhysioBank, PhysioToolkit, and PhysioNet: Components of a New Research Resource for Complex Physiologic Signals. Circulation. 2000 Jun 13;101(23):E215-20. doi: 10.1161/01.cir.101.23.e215.

[14] Biloborodova T, Scislo L, Skarga-Bandurova I, Sachenko A, Molga A, et al. Fetal ECG signal processing and identification of hypoxic pregnancy conditions in-utero, [J]. Mathematical Biosciences and Engineering, 2021; 18(4): 4919-4942. doi: 10.3934/mbe.2021250.

[15] Filatova A, Skarga-Bandurova I, Brezhniev E, Fahs M. Evaluating the Effectiveness of Electrocardiological Study Using Cardiological Decision Support Systems, CEUR Workshop Proceedings, 2020; 2711: 294-308. 